\title{
The effect of curcumin coated electrode on hydrogen production through water electrolysis
}

\author{
Willy Satrio ${ }^{1, *}$, Winarto $^{1}$, Sugiono $^{2}$, and I.N.G Wardana $^{1}$ \\ ${ }^{1}$ Dept. of Mechanical Engineering, Brawijaya University, 65144 Malang, Jawa Timur, Indonesia \\ ${ }^{2}$ Dept. of Industrial Engineering, Brawijaya University, 65144 Malang, Jawa Timur, Indonesia
}

\begin{abstract}
Hydrogen as the most cleanest energy source promise polutant free combustion. However, hydrogen production process still have negative impact on environment. Water electrolysis as one of hydrogen production process leave toxical gas residue and if the catalyst used in the process is metal catalyst it will leave metal waste which have potential to pollute soil and water. Curcumin as polyphenol antioxidant have potential as electrocatalytic agent due to its electron donor behavior. Curcumin loaded aluminum electrode coated by mechanical coating technique using water as solvent perform better as electrode compared to non-coated electrode. Curcumin coated cathode, anode and both electrode produce more hydrogen in shorter time than non-coated electrode. Curcumin electron donor increase the current density flows through the electrodes which is lift up the efficiency of water electrolysis process.
\end{abstract}

\section{Introduction}

Hydrogen is the cleanest and the most economically eficient fuel which promise pollutant free combustion [1]. Hydrogen can be obtained from water using water electrolysis technique. As a clean fuel hydrogen production process still have negative impact on environment. Water electrolysis requires noble metal catalysts and using acid or base solvent as conductance so the water can become more conductive. The usage of metal catalyst can leave metal residue which able to pollute soil and water. Acid or base solvent can produce toxical gas residue such as chlorine [2].

Curcumin electron donor potential has been observed as the antibacterial agent. The presence of curcumin on bacterial colloid can increase the zeta potential of the colloids and the combination of curcumin and graphene has the best performance to kill positive gram bacteria and break negative gram bacteria peptidoglycan cell wall in 8 hours duration using dim light photocatalysis technique [3]. The electron donor from the curcumin has potential to destabilize the electrons in water. Therefore, our study This research will apply curcumin electron donor potential to increase the efficieny of water electrolysis to create green water electrolysis technique.

Curcumin as the polyphenolic antioxidant have excess electron on its phenol aromatic structure [4]. Delocalized electron in aromatic structure create a resonance over time and the presence of oxygen in hydroxil functional group bend over the molecular orbital of aromatic ring since oxygen acting as electron scavenger $[4,5]$.

Curcumin act as reduction agent because its donate electron to stabilize free radical or other electron scavenger substance. Curcumin transfer the electron to free radical by creating electrostatic attraction to other molecules through hydroxyl functional group. Curcumin also able to create chelation with metal. Recent study report the curcumin chelated $\mathrm{Fe} 3+$ reduced to $\mathrm{Fe} 2+$ as the curcumin donate its electron to metal ion [4].

In this study, we report the electron donor effect of curcumin with the presence of delocalized electron able to increase current density in water electrolysis and reduce oxygen on electrode. We also investigate the effect of curcumin coating which able to help reduce the energy needed to break the water molecule in electrolysis.

\section{Material and Methods}

\subsection{Curcumin Coating}

Curcumin used in this experiment was obtained from $\mathrm{Jamu}^{\mathrm{TM}}$ brand curcumin extract powder which contains only curcumin. The curcumin coating process was using water medium [6]. The aluminum electrode was plunged into well stirred water-curcumin solution which stirred untill the water color changed to yellow and soaked for 12 hours in the solution in a closed manner to avoid contact with oxygen to prevent curcumin from oxidation. After the electrode dried, the electrolysis test was ready to conduct. Curcumin coating process shown in figure 1 (a) and the coated and uncoated electrode presents in figure 1(b) and 1(c) respectively. 


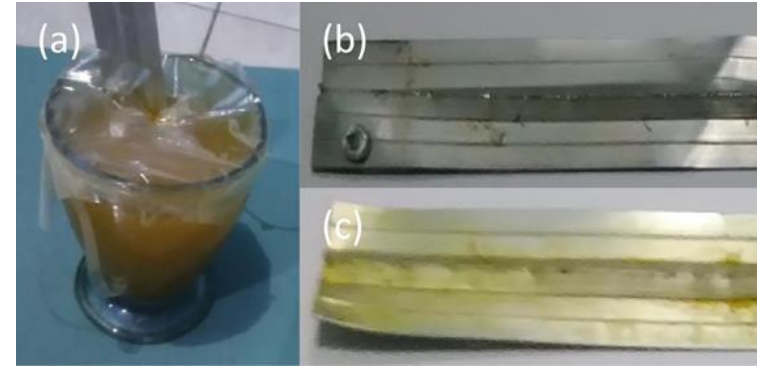

Fig. 1. (a) Curcumin Coating Process; (b) Uncoated Electrode; (c) Curcumin Coated Electrode

\subsection{Conductivity Testing}

The conductivity test was conducted using $12 \mathrm{~V}$ Battery as electricity source. The test was performed by pluging both cathode and anode to the coated rod and uncoated rod. 10 kohm resistor was placed on anode side due to the electron flow of battery from cathode to anode. The rod was connected to the battery using medium sized alligator clip. The multimeter was placed on both alligator clips to meassure the current flows. The electrical current data were measured in every 10 seconds. The apparatus setup of electrical conductivity is shown in figure 2 .

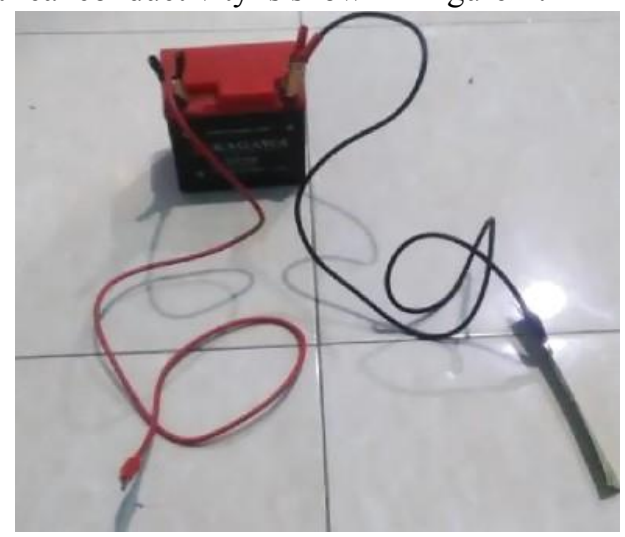

Fig. 2. Conductivity test Apparatus Setup

\subsection{Water Electrolysis Test}

The water electrolysis test was conducted in $250 \mathrm{ml}$ half filled glass leave out $125 \mathrm{~m} 1$ room for the gas and equipment. The source of electricity was $12 \mathrm{~V}$ battery and the cathode material was aluminum rod. The measurement was conducted using MQ8 hydrogen gas sensor interfaced with arduino board to the computer using load resistance of $10.31 \mathrm{kohm}$. The sensor placed beside the anode which attached to circular styrofoam as a cover. The apparatus design is shown in figure 3 .

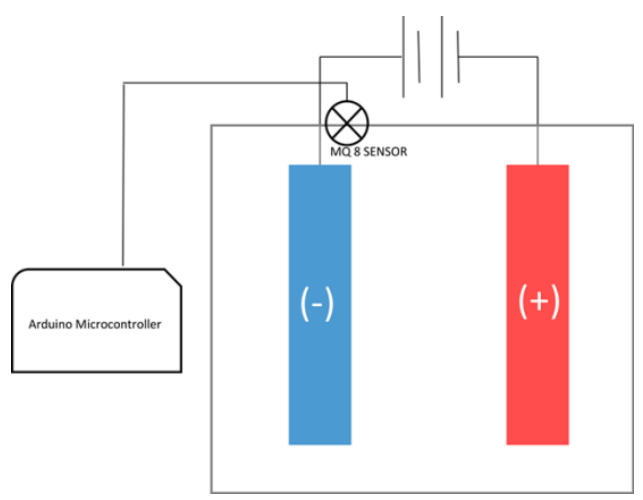

Fig. 3. Water Electrolysis test Apparatus Setup

\section{Result and Discussion}

\subsection{Curcumin Increase Electron Density}

The conductivity test was obtained by measuring the current flow pass through the material for 30 times. The material measured was noncoated rod, curcumin coated rod, activated carbon coated rod, and Curcuminactivated carbon coated rod. The result is shown in figure 4 .

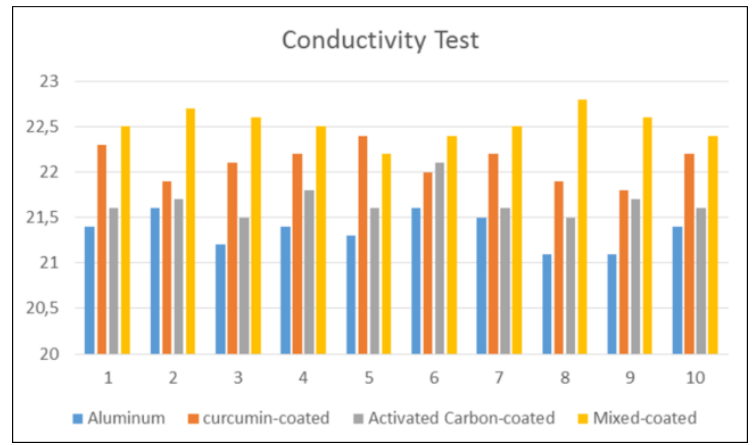

Fig. 4. Results of Conductivity Test

The electrical current flow in coated aluminum rod is higher than non-coated aluminum plate due to the electron donor from the aromatic ring to the material which causes the electron density on the material lattice getting higher and the current density which flow through the rod increased.

Increase of electron density also helps aluminum to reduce the oxygen attached on the material surface and free them [7]. The reduced amount of electron scavengers increase the electron mobility on the material surface so, the electrons can travel through material lattice freely without creating electrostatic attraction with oxygen nucleus which shown by reduced resistance factor and increased current [8]. There is no significance difference between activated carbon coated anode,curcumin coated anode, and mix coated anode conductivity as the average measurement of electrical current flow are $4.915 \mathrm{~A}, 4.873 \mathrm{~A}$, and $4.929 \mathrm{~A}$ respectively. This shows the symptom of electron donor 
effect and the increase of current density flow through material.

\subsection{Electron Investment Effect}

The curcumin coated electrode show the best performance of water electrolysis as it yields highest concentration of hydrogen gas in 5000 seconds. The uncoated electrode was outperformed by curcumin coated electrode with average difference of $1835 \mathrm{ppm}$ and 1840 ppm from curcumin coated electrode as shown in Figure 5.

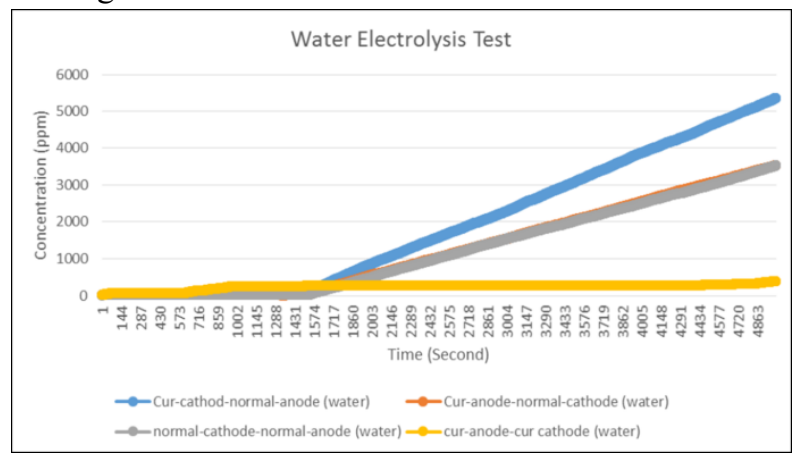

Fig. 5. Results of water electrolysis test

All curcumin coated aluminum electrode are able to outperform uncoated aluminum electrode due to the presence of aromatic ring. Delocalized electron movement in aromatic ring creates a magnetic field which interact with diamagnetic water molecule. The full outer shell of water molecular orbital reacts to magnetic field based on each electron spin orientation. The spin with same side of pole as the magnet will repulse as the spin with different pole pulled by the magnet causing the attracted electron to move towards magnetic field while the atom move in opposite direction as the nucleus electrostatic force stronger than magnetic field and by the help of the other spin that pull the left alone electron towards the nucleus, the left alone electron will be back and to its position by travel in some distance and vibrate due to the raise of kinetic energy because of the movement [5]. This will cause the water molecule to shake and widen its molecular orbital so, the hydrogen bond of water will be weaken because the electrostatic activity between atoms are weaken due to the increase of distance.

Curcumin coated cathode shows the best performance by achieve the highest concentration of yielded hydrogen in 5000 seconds. This happens due to the electron donor effect of curcumin. The excess electron of curcumin is contained in the aromatic ring. The hydroxyl functional group attached to curcumin aromatic ring allows curcumin to bind with oxygen on aluminum oxide [9]. The electrons are transferred and increase electron density of aluminum which adding the total charge transferred along anode to cathode. The increase of total charge will increase the total current density that flows in the system.

The fully coated electrode curcumin coated anode and cathode show lower hydrogen concentration result. The hydrogen yielded from both coated electrode only reach $392 \mathrm{ppm}$ in 5000 seconds. This happens due to the increase of potential in the water due to the electron donor activity. The electron donor activity of curcumin on cathode is shown in Figure 6.

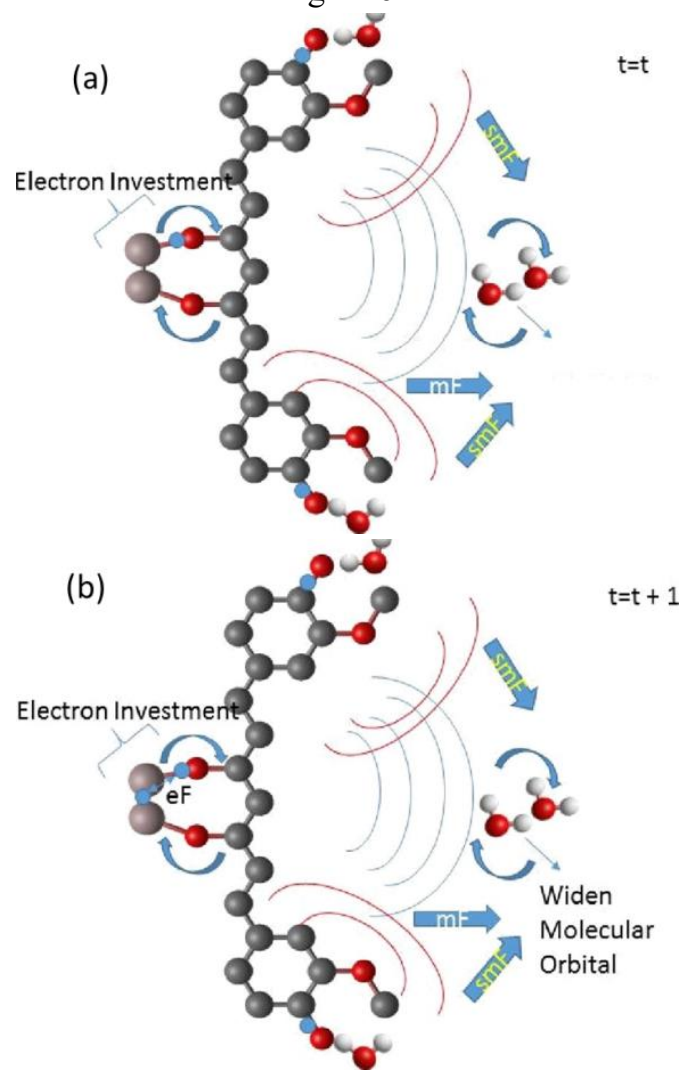

Fig. 6. chelation of aluminum electrode : (a) initial state, (b) at next time step.

The electron donor activity of curcumin at initial state is started by chelation of electrode with curcumin. Binded aluminum electrode on creating new molecular orbital for electron to orbit which is creating a room for electron to orbit freely such in aromatic ring. At subsequence time step, the electron which flow through aluminum cathode generates electrostatic interaction by repulse the free electron in chelated region resulting in electrostatic force that push the electron stronger than the potential applied to electrode. This electron investment phenomenon explain why the water electrolysis test result on figure 4 showing stable pattern at first and then generate incremental pattern instantaneously. 


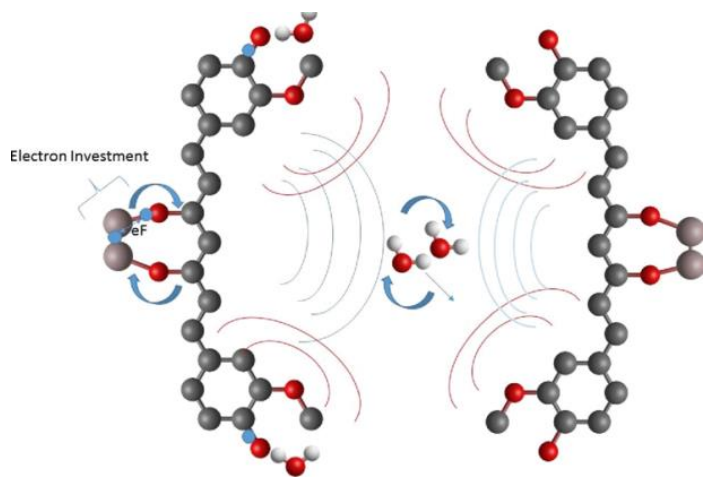

Fig. 7. Equal phase magnetic force on both coated electrode

The magnetic force plays role to raise kinetic energy of water. However, the induced magnetic field by both electrode are in equal phase when both electrode coated by curcumin as shown at figure 5 . This equal phase proven by the hydrogen concentration released of both coated electrode during the electrolysis which shown at figure 4, yields lowest hydrogen concentration and inhibit the electrolysis process.

Compared to previous research in water electrolysis using aluminum electrode, curcumin coated electrode have better performances. Hydrolytic reaction of aluminum foil hydrolysis in alkalized water only able to produce $1300 \mathrm{ppm}$ of hydrogen gas in 5 hours [10]. More over this technique is less friendly to the environment due to the potential of soil toxication by alkalized water waste [11]. While in our study which is more environmental friendly, by applying organic substance, curcumin coated to the electrode is able to produce $5367 \mathrm{ppm}$ in around 1.5 hours. This is around ten folds larger. The recent technology of water electrolysis using proton exchange membrane (PEM) with $\mathrm{pt} / \mathrm{c}$ electrode is still the most efficient and effective hydrogen production technique using water electrolysis. However, the capital cost needed to build PEM hydrogen production system is the highest among other water electrolysis technique [12]. Curcumin coated electrode can be used as an alternative solution to build cheaper but effective electrolyzer using organic substance.

\section{Conclusions}

This paper has shown the alternative and more sustainable way to produce hydrogen through water electrolysis using antioxidant catalyst and show the potential of curcumin as one of polyphenolic antioxidant as an organic catalyst. This approach leads to the metal free water electrolysis and not needed any noble material. This technique is possible because of electron donor effect by the delocalized electron contained in aromatic ring transferred to the electrode material and increase the current density. The electron donor effect of curcumin phenol ring structure coated on cathode reported in this study for the first time and lead to the metal free sustainable organic catalyst for water electrolysis.

This study is made possible by Indonesia Ministry of Research and Higher Education (KEMENRISTEKDIKTI) under PMDSU program with grant number [147/SP2H/LT/DRPM/2018].

\section{References}

1. J. A. Turner, Science (80-. ). 305, 972 (2004)

2. L. Yang, W. Liu, Z. Zhang, X. Du, J. Gong, L. Dong, and Y. Deng, Electrochim. Acta 246, 1163 (2017)

3. Z. M. Marković, D. P. Kepić, D. M. Matijašević, V. B. Pavlović, S. P. Jovanović, N. K. Stanković, D. D. Milivojević, Z. Spitalsky, I. D. HolclajtnerAntunović, D. V. Bajuk-Bogdanović, M. P. Nikšić, and B. M. Todorović Marković, RSC Adv. 7, 36081 (2017)

4. Y. X. Chen, A. Lavacchi, H. A. Miller, M. Bevilacqua, J. Filippi, M. Innocenti, A. Marchionni, W. Oberhauser, L. Wang, and F. Vizza, Nat. Commun. 5, (2014)

5. A. D. Wright, C. Verdi, R. L. Milot, G. E. Eperon, M. A. Pérez-Osorio, H. J. Snaith, F. Giustino, M. B. Johnston, and L. M. Herz, Nat. Commun. 7, (2016)

6. S. Çakir, E. Biçer, and E. Y. Arslan, Croat. Chem. Acta 88, 105 (2015)

7. O. Leenaerts, B. Partoens, and F. M. Peeters, Phys. Rev. B - Condens. Matter Mater. Phys. 77, (2008)

8. M. A. Molina-García and N. V. Rees, Appl. Catal. A Gen. 553, 107 (2018)

9. M. A. Atanga, F. Rezaei, A. Jawad, M. Fitch, and A. A. Rownaghi, Appl. Catal. B Environ. 220, 429 (2018)

10. C. Y. Ho, Int. J. Hydrogen Energy 42, 19622 (2017)

11. C. Gao, Q. Zhao, and L. Jiang, Proc. Natl. Acad. Sci. U. S. A. 112, 2931 (2015)

12. O. Schmidt, A. Gambhir, I. Staffell, A. Hawkes, J. Nelson, and S. Few, Int. J. Hydrogen Energy 42, 30470 (2017) 